\title{
Adjoint sensitivity analysis of an ultrawideband antenna
}

M. B. Stephanson, D. A. White

August 1, 2011

IEEE Antennas and Propagation Society Toronto, Canada July 11, 2010 through July 17, 2010 
This document was prepared as an account of work sponsored by an agency of the United States government. Neither the United States government nor Lawrence Livermore National Security, LLC, nor any of their employees makes any warranty, expressed or implied, or assumes any legal liability or responsibility for the accuracy, completeness, or usefulness of any information, apparatus, product, or process disclosed, or represents that its use would not infringe privately owned rights. Reference herein to any specific commercial product, process, or service by trade name, trademark, manufacturer, or otherwise does not necessarily constitute or imply its endorsement, recommendation, or favoring by the United States government or Lawrence Livermore National Security, LLC. The views and opinions of authors expressed herein do not necessarily state or reflect those of the United States government or Lawrence Livermore National Security, LLC, and shall not be used for advertising or product endorsement purposes. 


\title{
Adjoint Sensitivity Analysis of an Ultrawideband Antenna
}

\author{
Matthew Stephanson ${ }^{(1)}$, Daniel A. White*(1) \\ Lawrence Livermore National Laboratory, Livermore, CA, USA \\ E-mail: white37@llnl.gov
}

\section{Introduction}

The frequency domain finite element method using $H($ curl)-conforming finite elements is a robust technique for full-wave analysis of antennas. As computers become more powerful, it is becoming feasible to not only predict antenna performance, but also to compute sensitivity of antenna performance with respect to multiple parameters. This sensitivity information can then be used for optimization of the design or specification of manufacturing tolerances. In this paper we review the Adjoint Method for sensitivity calculation, and apply it to the problem of optimizing a Ultrawideband antenna.

\section{Adjoint Method}

The goal of sensitivity analysis is to determine how the answer to a problem changes when the inputs change slightly. Mathematically, we are given a set of parameters $\mathbf{p}=\left\{p_{1}, p_{2}, \ldots, p_{N}\right\}$ that describe a problem (e.g., a ODE or integral equation) leading to a solution $\mathbf{u}$. Also given is a response functional $R(\mathbf{p}, \mathbf{u})$ that depends on the solution and may also explicitly depend on the parameters. Sensitivity analysis consists of choosing a baseline set of parameters $\mathbf{p}^{\mathbf{0}}$ and calculating

$$
\nabla R=\left.\left(\frac{d R}{d p_{1}}, \frac{d R}{d p_{2}}, \ldots, \frac{d R}{d p_{N}}\right)\right|_{\mathbf{p}=\mathbf{p}^{\mathbf{0}}} .
$$

Note that $d R / d p_{1}$ refers to the total derivative, while $\partial R / \partial p_{1}$ is reserved for the derivative with respect to the explicit $p_{1}$ dependence. In other words, using the chain rule,

$$
\frac{d R}{d p_{1}}=\frac{\partial R}{\partial p_{1}}+\frac{\partial R}{\partial \mathbf{u}} \frac{\partial \mathbf{u}}{\partial p_{1}}
$$

In the following, for simplicity, we consider the sensitivity with respect to one of the variables. Also, adopting matrix terminology, we assume that $\mathbf{u}$ comes from a linear system $A \mathbf{u}=\mathbf{b}$ and the response has the form $R=\mathbf{c} \cdot \mathbf{u}$.

With the previous assumptions, the sensitivity equation has the form [2]

$$
\frac{d R}{d p}=\mathbf{c}^{\prime} \cdot \mathbf{u}+\mathbf{c} \cdot A^{-1}\left(\mathbf{b}^{\prime}-A^{\prime} \mathbf{u}\right)
$$

where a prime denotes partial differentiation with respect to the parameter $p$. The forward sensitivity method consists of finding $A^{-1}\left(\mathbf{b}^{\prime}-A^{\prime} \mathbf{u}\right)$ for each parameter, 


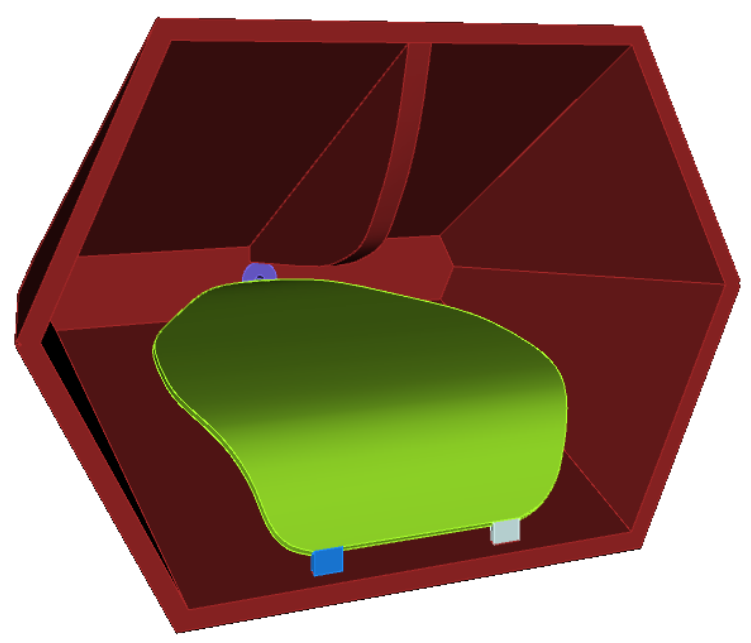

Figure 1: Ultrawideband antenna example. The aperture is approximately 3.2 inches wide and 2.2 inches tall. The two blocks below the tongue represent $100 \Omega$ resistors.

requiring $N$ additional matrix solutions. If the number of parameters is large, this procedure can be expensive. Instead, introduce the adjoint variable $\boldsymbol{\psi}$, defined by

$$
A^{H} \boldsymbol{\psi}=\mathbf{c} .
$$

Then the second term in (3) becomes

$$
\mathbf{c} \cdot A^{-1}\left(\mathbf{b}^{\prime}-A^{\prime} \mathbf{u}\right)=\boldsymbol{\psi} \cdot\left(\mathbf{b}^{\prime}-A^{\prime} \mathbf{u}\right) .
$$

This is the adjoint method, and requires only one additional matrix solve, to find $\boldsymbol{\psi}$, plus $N$ matrix-vector multiplications.

To implement the adjoint method we built upon an existing code framework called EMSolve. In simple terms, the matrix equation $A \mathbf{x}=\mathbf{b}$ is the $H($ curl $)$-conforming finite element discretization of

$$
\nabla \times \frac{1}{\mu_{r}} \nabla \times \mathbf{E}-k_{0}^{2}\left(\varepsilon_{r}-j \frac{\sigma}{\omega \varepsilon_{0}}\right) \mathbf{E}=-j \omega \mu_{0} \mathbf{J}^{i m p}
$$

The basis functions, quadrature rules, matrix assembly, and matrix solution has been described previously [3]. In our implementation the partial derivatives $A^{\prime}$ are computed exactly using automatic differentiation [1]. The responses $R=\mathbf{c} \cdot \mathbf{u}$ are line integrals of $\mathbf{E}$ along an arbitrary path. This encompasses, for example, voltage on a transmission line and (with minor modifications) $S$ parameters. By reformulating the problem in terms of the magnetic field, the current on a conductor can be calculated in a likewise manner.

\section{Ultra-wideband Antenna Analysis}

The antenna in Fig. 1 has been analyzed. This is a horn antenna with a desired frequency operation from $2 \mathrm{GHz}$ to $5 \mathrm{GHz}$. The parameters are the conductivity in the two resistive blocks, as well as the permittivity, to explore the effects of 

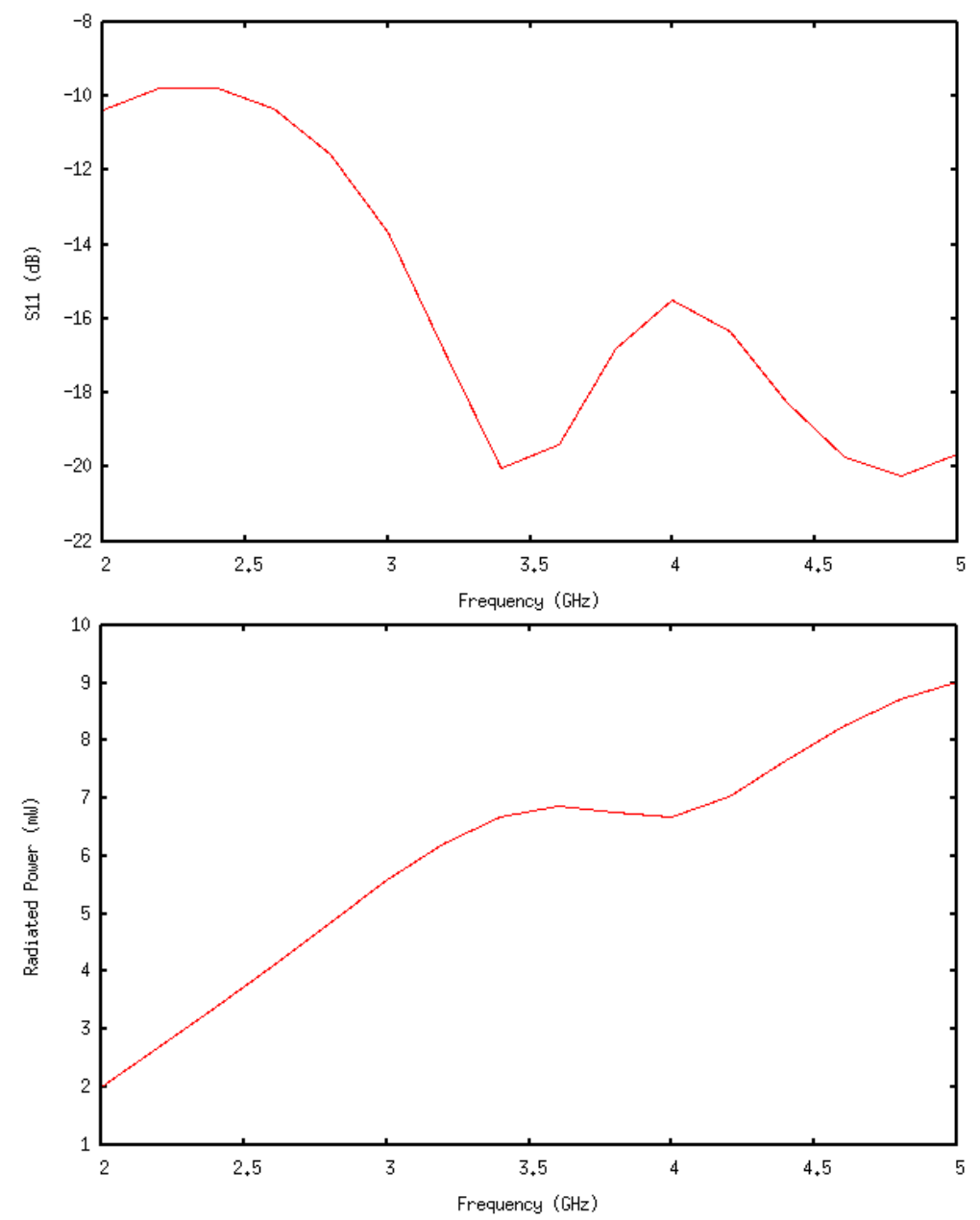

Figure 2: The top plot is the antenna return loss, S11. The bottom plot is the radiated power for $10 \mathrm{~mW}$ input.

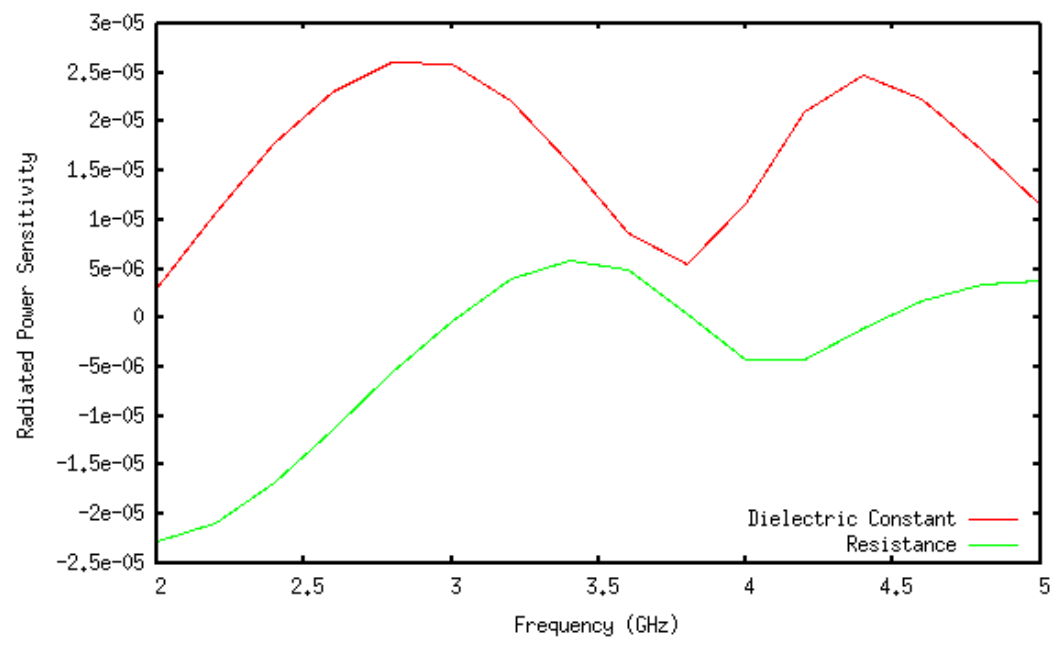

Figure 3: Sensitivity of radiated power w.r.t resistance (lower curve) and capacitance (upper curve). 
capacitive loading. The antenna is fed by a $1 \mathrm{~V}$ wave on a $50 \Omega$ coaxial line. In addition to a line integral response, in the form of the voltage on the transmission line, this problem introduces another type of response, the ohmic loss which is a quadratic form $\mathbf{E}^{H} M \mathbf{E}$, where $\mathrm{M}$ is a finite element mass matrix involving the electrical conductivity. For sensitivity purposes, we require an analog of (3) for this new type of response. Assuming that $M$ is Hermitian, the result is

$$
\begin{aligned}
R & =\frac{1}{2} \mathbf{u}^{H} M \mathbf{u} \\
\frac{d R}{d p} & =\frac{1}{2} \mathbf{u}^{H}\left(M^{\prime} \mathbf{u}+M \mathbf{x}^{\prime}\right)+\frac{1}{2}\left(\mathbf{x}^{\prime}\right)^{H} M \mathbf{u} \\
& =\frac{1}{2} \mathbf{u}^{H} M^{\prime} \mathbf{u}+\frac{1}{2} \mathbf{u}^{H} M A^{-1}\left(\mathbf{b}^{\prime}-A^{\prime} \mathbf{u}\right)+\frac{1}{2}\left(\mathbf{b}^{\prime}-A^{\prime} \mathbf{u}\right)^{H} A^{-H} M \mathbf{u} \\
& =\frac{1}{2} \mathbf{u}^{H} M^{\prime} \mathbf{u}+\boldsymbol{\psi}^{H}\left(\mathbf{b}^{\prime}-A^{\prime} \mathbf{u}\right)+\left(\mathbf{b}^{\prime}-A^{\prime} \mathbf{u}\right)^{H} \boldsymbol{\psi},
\end{aligned}
$$

where $A^{H} \boldsymbol{\psi}=\frac{1}{2} M \mathbf{u}$ defines the adjoint solution in this case. With the voltage on the transmission line, $V$, and the power in each resistor, $P_{1}$ and $P_{2}$, several other quantities were derived via post processing:

$$
\begin{aligned}
\Gamma & =V-1 \\
P_{\text {rad }} & =\frac{1-|\Gamma|^{2}}{2 Z_{0}}-P_{1}-P_{2},
\end{aligned}
$$

where $Z_{0}=50 \Omega$ is the characteristic impedance of the feed line, $\Gamma$ is the reflection coefficient, and $P_{\text {rad }}$ is the radiated power.

Figure 2 shows the radiated power over $2-5 \mathrm{GHz}$. Interestingly, the return loss was quite small over the entire band, hence the resistors dissipate most of the power at low frequencies. The sensitivities of radiated power with respect to the resistance and dielectric constant of the resistor blocks are shown in Fig. 3. The sensitivity with respect to resistance is negative at low frequencies and closer to zero at higher frequencies, therefore the low frequency gain can be improved by decreasing the resistance. The sensitivity with respect to dielectric constant is positive, therefore radiated power can also be improved by adding capacitors in parallel to the resistors. This illustrates how adjoint sensitivity analysis can be used to optimize the value of lumped elements in a broadband antenna, at a computational cost of only one additional linear solve.

\section{References}

[1] R. A. Bartlett, D. M. Gray, and E. T. Phillips. Automatic differentiation of $\mathrm{C}++$ codes for large-scale scientific computing. Lec. Notes Comp. Sci., 3994:525 $-532,2006$.

[2] Dan G. Cacuci. Sensitivity and Uncertainty Analysis, Volume 1. Chapman \& Hall/CRC, 2003.

[3] P. Castillo, R. Rieben, and D. White. FEMSTER: An object orienetd class library of higher-order discrete differential forms. ACM Trans. Math. Soft., 31(4):425-457, 2005.

This work performed under the auspices of the U.S. Department of Energy by Lawrence Livermore National Laboratory under Contract DE-AC52-07NA27344. 